\title{
Doxycycline in Eradication Therapy of Helicobacter pylori - A Systematic Review and Meta-Analysis
}

\author{
Yaron Niv \\ Department of Gastroenterology, Rabin Medical Center, Tel Aviv University, Tel Aviv, Israel
}

\section{Key Words}

Doxycycline $\cdot$ Helicobacter pylori · Eradication · Gastritis

\begin{abstract}
Background: Since resistance of Helicobacter pylori is developing very fast all over the world, new treatment regimens for eradication are urgently needed. Aim: To compare eradication success rate of $H$. pylori treatment regimens with and without doxycycline. Methods: English medical literature searches were conducted for regimens including doxycycline for eradication of $H$. pylori. Searches were performed up to August 31, 2015, using MEDLINE, PubMed, EMBASE, Scopus and CENTRAL. Meta-analysis was performed by using comprehensive meta-analysis software. Pooled ORs and 95\% Cls were calculated comparing treatment regimens for eradication of $H$. pylori infection with and without doxycycline. Results: The OR for eradication success rate in a fixed model was in favor for treatment regimens with doxycycline: $1.292,95 \% \mathrm{Cl} 1.048-1.594, \mathrm{p}=0.016$. There was no significant heterogeneity in the included studies: $Q=15.130$, d.f. $(Q)=8, I^{2}=47.126, p>0.10$. When treatment regimens with doxycycline were compared only with treatment regimens with tetracycline, no significant difference was found in eradication success rate: $\mathrm{OR} 0.95,95 \% \mathrm{Cl} 0.68-1.32, \mathrm{p}=0.77$. But when treatment regimens with doxycycline were com-
\end{abstract}

pared with treatment regimens without tetracycline, the OR in favor of doxycycline was even higher: OR $1.59,95 \% \mathrm{Cl}$ 1.21-2.09, $\mathrm{p}<0.001$. Conclusion: In this meta-analysis, we confirmed doxycycline efficiency in the eradication of $H$. pylori. Thus, triple, quadruple or even high dose dual therapy with regimens containing doxycycline should be considered.

(c) 2016 S. Karger AG, Basel

\section{Introduction}

Since resistance of Helicobacter pylori is developing very fast in many countries all over the world, new treatment regimens for eradication are urgently needed. Amoxicillin is a very effective part of every regimen, and resistance to this antibiotic is very rare. Patients, who are allergic to penicillin, cannot take amoxicillin and a substitute is needed. Tetracycline has a similar therapeutic range as amoxicillin, and may be active against $H$. $p y$ lori. Substitution of doxycycline for tetracycline HCL offers advantages of less frequent dosing and extra renal excretion. Doxycycline was first demonstrated to be effective drug against $H$. pylori in 1992, with a MIC $0.25-$ $8.0 \mu \mathrm{g} / \mathrm{ml}$ [1]. The first reports of doxycycline substitute for tetracycline were disappointing in this regard $[2,3]$.

\section{KARGER}

E-Mail karger@karger.com www.karger.com/dig (c) 2016 S. Karger AG, Base

0012-2823/16/0932-0167\$39.50/0
Professor Yaron Niv, MD, AGAF, FACG

Department of Gastroenterology

Rabin Medical Center, 39 Jabotinski Street

Petach Tikva 49100 (Israel)

E-Mail yniv@ clalit.org.il 
But the results of the paper by Realdi et al. [3] were somewhat confusing because of the high resistance rate not only to doxycycline (33.3\%), but also to amoxicillin (26\%). In contrast, Usta et al. [4] found a higher eradication rate in a second-line quadruple therapy including doxycycline than 2 first-line triple therapies in children, 64.6 versus 60.5 and $55.8 \%$, respectively. Recently, a combination of esomeprazole, levofloxacin and doxycycline was tried as a third-line therapy with a low eradication efficacy that was somewhat enhanced with probiotics [5].

In this meta-analysis, we looked at studies that compared the eradication success rate of $H$. pylori treatment regimens with and without doxycycline. A high rate of successful eradication will give legitimation to use doxycycline instead of amoxicillin in cases of penicillin allergy or as a rescue quadruple therapy.

\section{Methods}

\section{Search Strategy}

English medical literature searches were conducted for treatment regimens which included doxycycline for eradication of $H$. pylori. Searches were performed through August 31, 2015, using MEDLINE, PubMed, EMBASE, Scopus and CENTRAL. Search terms were: ('doxycycline'(MeSH Terms) OR 'doxycycline'(All Fields)) AND ('Helicobacter pylori'(MeSH Terms) OR ('Helicobacter'(All Fields) AND 'pylori'(All Fields)) OR 'Helicobacter pylori'(All Fields)). Hand searches of articles were performed after the initial search, and included article bibliography. Only fully published human studies in English were included (fig. 1).

\section{Study Selection}

Case-control studies comparing treatment regimens for eradication of $H$. pylori, with and without doxycycline, were included. H. pylori infection should be diagnosed with ${ }^{13} \mathrm{C}$-urea breath test, stool antigen, rapid urease test or histology and successful eradication with one of the same tests, at least a month after treatment, and at least 2 weeks without antibiotics, bismuth or proton pump inhibitor therapy. We selected only randomized, prospective, controlled studies that used intention to treat method to express the results.

\section{Data Extraction}

Successful eradication rates were extracted for treatment regimens with and without doxycycline. In studies where sub-studies were performed, such as comparison of first and second regimens, gender, treatment specification such as triple, quadruple, concomitant or sequential regimens, their results were compared in innate studies.

Meta-analysis was performed by using comprehensive metaanalysis software (version 3, Biostat Inc., Englewood, N.J., USA). Pooled ORs and 95\% CIs were calculated comparing eradication success rate in individual studies by using the fixed effects model.

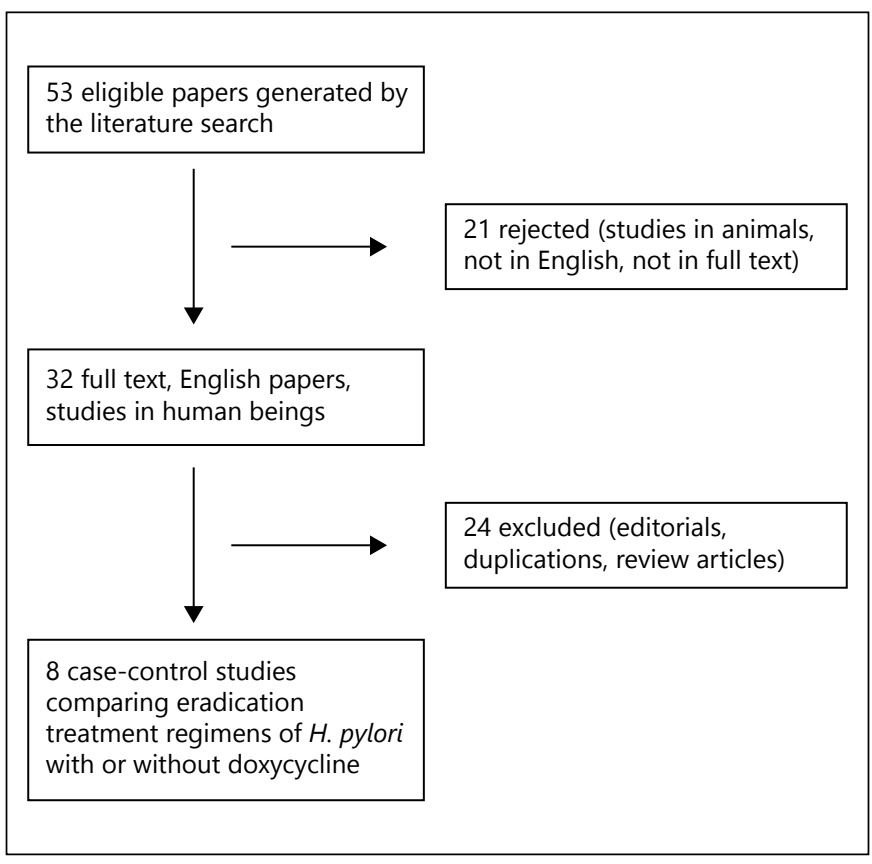

Fig. 1. Flow chart of the articles identified for the meta-analysis.

Heterogeneity between studies was evaluated using the Cochran $\mathrm{Q}$ test, and it was considered to be present if the $\mathrm{Q}$ test $\mathrm{p}$ value was $<0.10$. $\mathrm{I}^{2}$ statistic was used to measure the proportion of inconsistency in individual studies, with $\mathrm{I}^{2}>50 \%$ representing substantial heterogeneity. We also calculated a potential publication bias.

\section{Results}

All together we found 53 eligible studies. Twenty-one studies were rejected because of language, for being performed in animals or not having full text. Twenty-four papers were excluded being editorials, review articles or because of duplications. We were left with 8 case-control studies from 8 countries, comparing eradication treatments of $H$. pylori with or without doxycycline, that fulfilled the inclusion criteria, published till August 31, 2015 [2, 3, 6-11] (fig. 1; table 1). There are 15 sub-studies (innate studies, stratifying data according to treatment regimen or duration). In the study from Texas [3], $5 \mathrm{sub-}$ studies have been performed, comparing the same doxycycline regimen with 5 different regimens without doxycycline. The studies from Italy, Iran and New York $[6,8,10]$ include 2 sub-studies each. Since Texas and New York are big states in the USA, with different demographic characteristics (many black people in New York, 


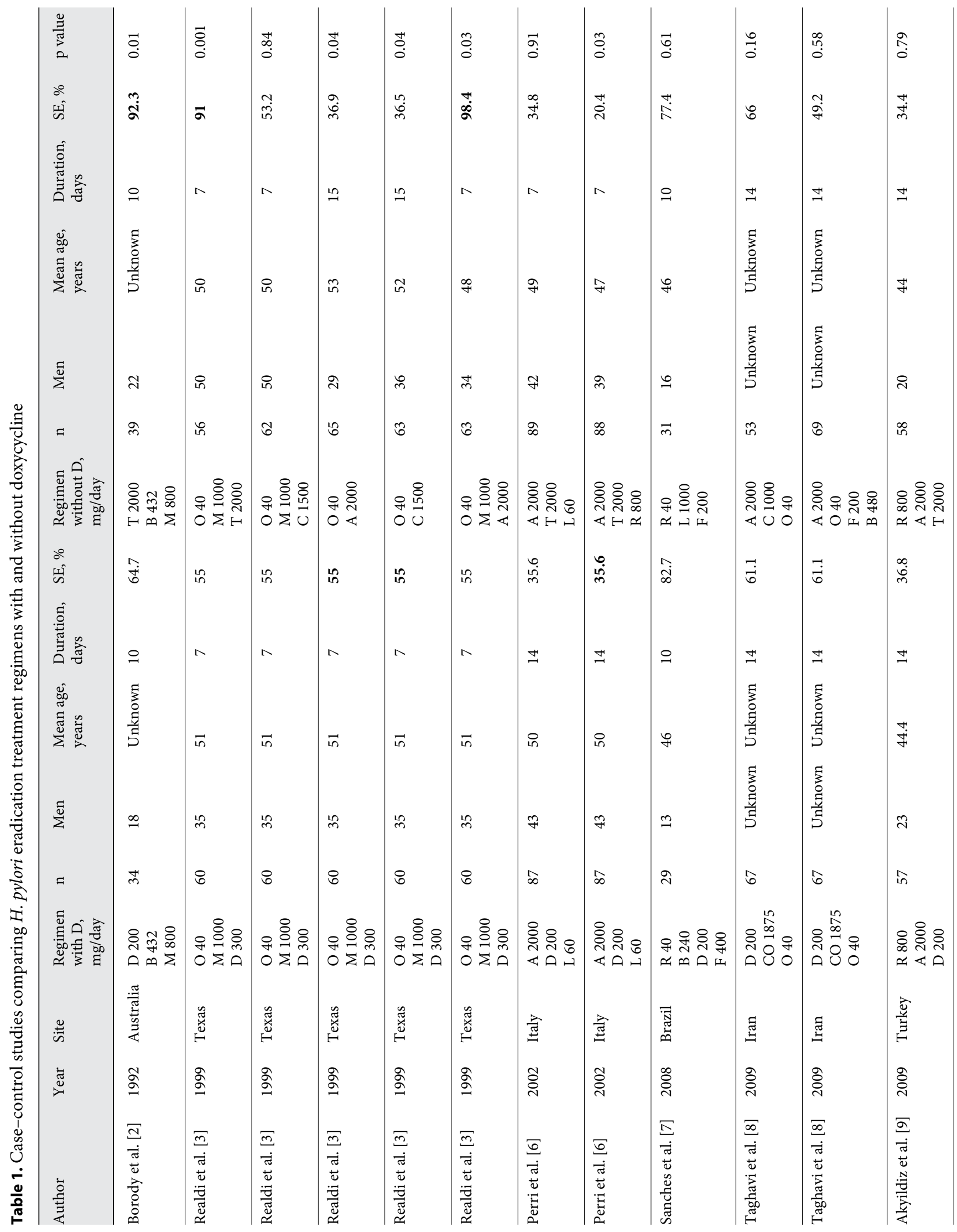




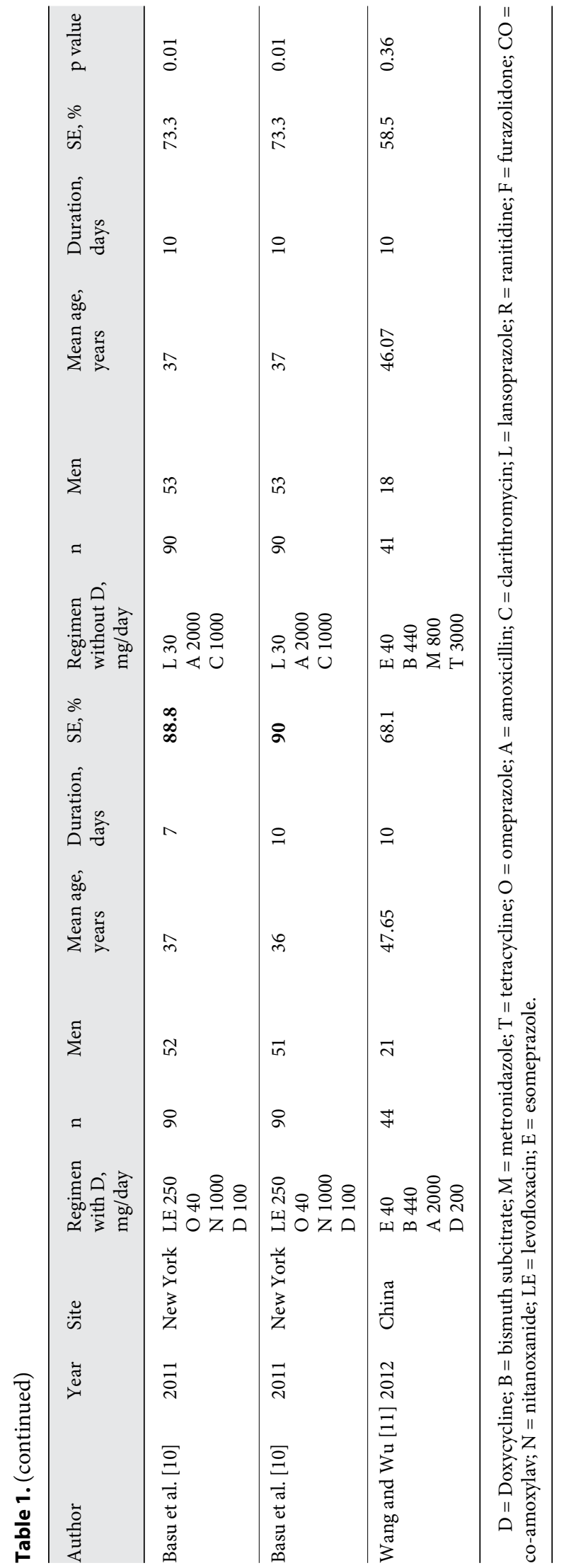

and many Hispanic in Texas), we preferred to separate the results. The regimens used were different, and the studies from Texas and New York were published 12 years apart.

Out of 15 sub-studies, the results of 8 reached significance (table 1) $[2,3,6,10]$. In 5 sub-studies, treatment regimens with doxycycline had significantly higher eradication rate than treatment regimens without doxycycline (table 1) $[3,6,10]$. In 3 sub-studies, treatment regimens without doxycycline have done better (table 1) [2, 3]. In 6 sub-studies, treatment regimens with doxycycline were compared with treatment regimens containing tetracycline (table 1) [2, 3, 6, 9, 11]. In 2 sub-studies tetracycline was better $[2,3]$, in 1 substudy doxycycline was better [6] and in the other no difference between them was demonstrated (table 1) $[6,9$, 11 .

The OR for eradication rate in a fixed model was in favor for treatment regimens with doxycycline: 1.292, 95\% CI 1.048-1.594, $\mathrm{p}=0.016$ (fig. 2). Funnel plot denies a significant publication bias (fig. 3). There was no significant heterogeneity in the included studies: $Q=15.130$, d.f. $(\mathrm{Q})=8, \mathrm{I}^{2}=47.126, \mathrm{p}>0.10$.

When only treatment regimens with doxycycline were compared with treatment regimens with tetracycline, no significant difference was found in eradication success rate: OR 0.95, 95\% CI 0.68-1.32, $\mathrm{p}=0.77$ (fig. 2a). But when treatment regimens with doxycycline were compared with treatment regimens without tetracycline, the OR in favor of doxycycline was even higher: OR 1.59, 95\% CI 1.21-2.09, $\mathrm{p}<0.001$.

\section{Discussion}

Doxycycline was clinically developed in the early $1960 \mathrm{~s}$ and approved by the FDA in 1967 [12]. It belongs to the tetracycline group of antibiotics, with improved stability and pharmacological efficacy. Tetracycline and doxycycline have therapeutic range similar to penicillin, thus, theoretically could replace amoxicillin in the treatment regimen for $H$. pylori. This may be especially important in patients allergic to penicillin. Both drugs, tetracycline and doxycycline, act systemically after absorption from the upper gastrointestinal tract. The main advantage of doxycycline over tetracycline is its longer activity, and it can be taken twice or once a day. The peak concentration of both drugs is similar, but in the case of doxycycline the time to peak concentration is shorter, and half life is significantly longer [13]. 


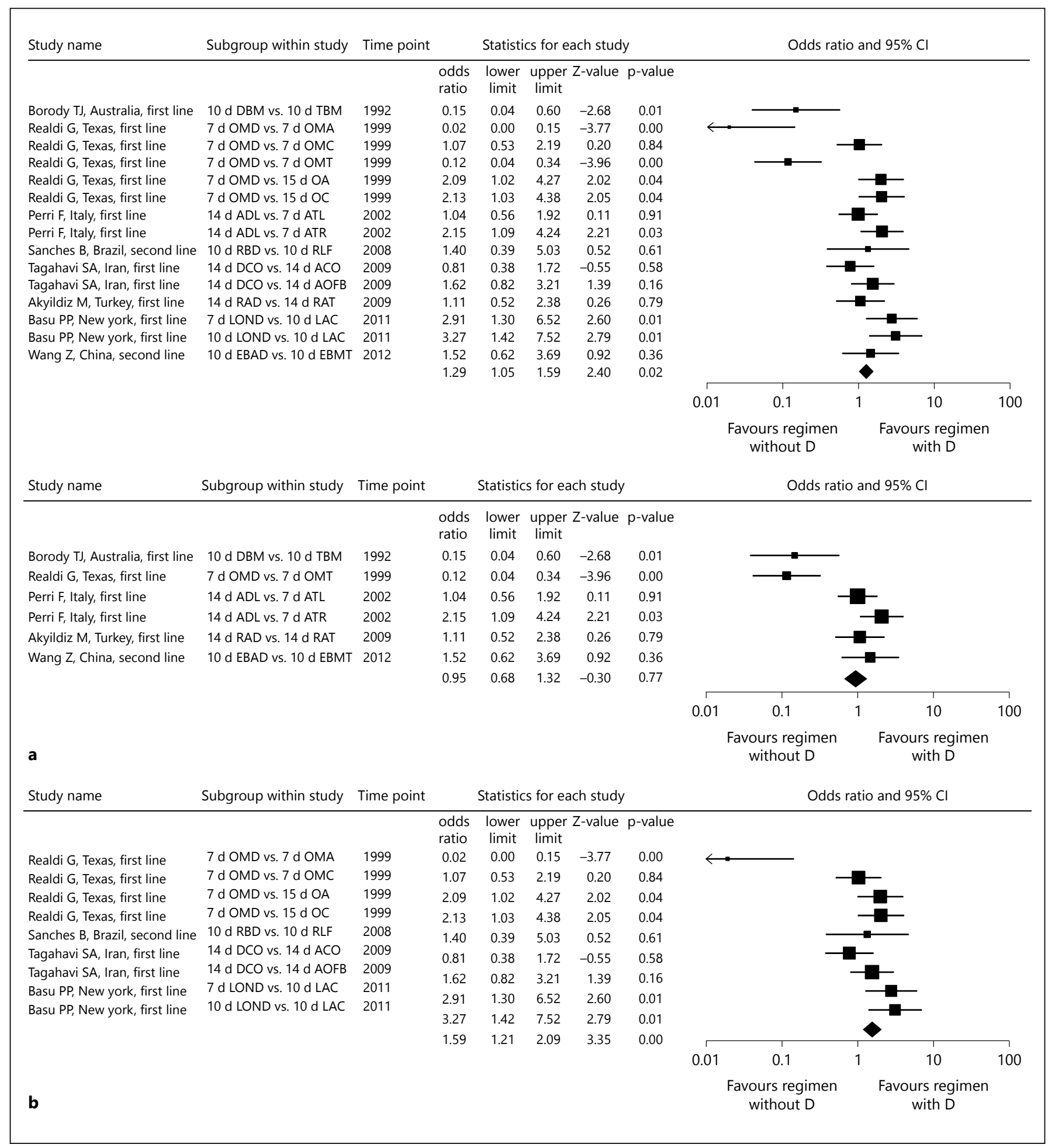

Fig. 2. Meta-analysis of studies comparing eradication success rate of treatment regimens, with and without doxycycline (8 papers and 15 studies). a Meta-analysis of studies comparing eradication success rate of treatment regimens with doxycycline and those with tetracycline ( 5 papers and 6 studies). b Meta-analysis of studies comparing eradication success rate of treatment regimens with doxycycline and those without tetracycline (4 papers and 9 studies). $\mathrm{D}=$ Doxycycline; $\mathrm{B}=$ bismuth subcitrate; $\mathrm{M}=$ metronidazole; $\mathrm{T}$ = tetracycline; $\mathrm{O}=$ omeprazole; $\mathrm{A}=$ amoxicillin; $\mathrm{C}=$ clarithromycin; $\mathrm{L}=$ lansoprazole; $\mathrm{R}=$ ranitidine; $\mathrm{F}$ = furazolidone; $\mathrm{CO}=$ co-amoxylav; $\mathrm{N}$ = nitanoxanide; $\mathrm{LE}=$ levofloxacin; $\mathrm{E}=$ esomeprazole; $\mathrm{d}=$ days. 
Fig. 3. Funnel plot for publication bias.

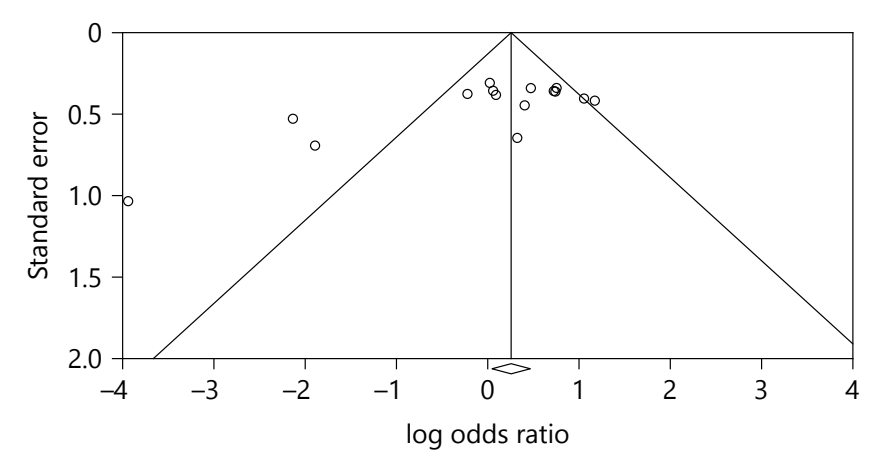

In this meta-analysis, we confirmed doxycycline efficiency in the eradication of $H$. pylori. Treatment regimens containing doxycycline had a similar eradication success rate as regimens containing tetracycline, but better than those without a representative of the tetracycline family of antibiotics. When treatment regimens with tetracycline HCL were excluded, the OR for doxycycline containing regimens reached 1.59 and is very significant $(\mathrm{p}<$ $0.001)$.

I believe that according to this meta-analysis, triple, quadruple or even high dose dual therapy with regimens containing doxycycline will be considered as a therapeutic option. This observation is particularly important in the era of drug resistance and the increasing need for alternative eradication regimens and rescue therapy [14].

This study is limited by the lack of many trials with treatment regimens containing doxycycline, reporting only 8 case-control studies. In addition, some of the studies described first-line therapy and some rescue therapy, thus eradication success varied and was $<85 \%$. Thus, the results should be taken with caution, and more randomized controlled studies are needed for final conclusions.

\section{Summary}

In this meta-analysis, we looked at studies that compared the eradication success rate of $H$. pylori treatment regimens with and without doxycycline. The OR for eradication rate in a fixed model was in favor for treatment regimens with doxycycline: 1.292 , 95\% CI 1.048-1.594, $\mathrm{p}=0.016$. There was no significant heterogeneity in the included studies: $\mathrm{Q}=15.130$, d.f. $(\mathrm{Q})=8, \mathrm{I}^{2}=47.126$, $\mathrm{p}$ > 0.10 . When treatment regimens with doxycycline were compared only with treatment regimens with tetracycline, no significant difference was found in eradication success rate. But, when treatment regimens with doxycycline were compared with treatment regimens without tetracycline, the OR in favor of doxycycline was even higher. Thus, triple, quadruple or even high dose dual therapy with regimens containing doxycycline should be considered.

\section{Disclosure Statement}

Professor Y. Niv has no conflict of interest to declare.

\section{Data Sharing Statement}

Technical appendix, statistical code and data set available from the corresponding author at nivyaron80@gmail.com. No additional data are available.
References
1 Loo VG, Sherman P, Matlow AG: Helicobacter pylori infection in a pediatric population: in vitro susceptibilities to omeprazole and eight antimicrobial agents. Antimicrob Agents Chemother 1992;36:1133-1135.

2 Borody TJ, George LL, Brandl S, Andrews P, Lenne J, Moore-Jones D, Devine M, Walton M: Helicobacter pylori eradication with doxycycline-metronidazole-bismuth subcitrate triple therapy. Scand J Gastroenterol 1992;27: 281-284.

3 Realdi G, Dore MP, Piana A, Atzei A, Carta M, Cugia L, Manca A, Are BM, Massarelli G, Mura I, Maida A, Graham DY: Pretreatment antibiotic resistance in Helicobacter pylori infection: results of three randomized controlled studies. Helicobacter 1999;4:106112. 
4 Usta Y, Saltik-Temizel IN, Demir H, Uslu N, Ozen H, Gurakan F, et al: Comparison of short- and long-term treatment protocols and the results of second-line quadruple therapy in children with Helicobacter pylori infection. J Gastroenterol 2008;43:429-433.

5 Paoluzi OA, Del Vecchio Blanco G, Visconti E, Coppola M, Fontana C, Favaro M, Pallone F: Low efficacy of levofloxacin-doxycyclinebased third-line triple therapy for Helicobacter pylori eradication in Italy. World J Gastroenterol 2015;21:6698-6705.

6 Perri F, Festa V, Merla A, Quitadamo M, Clemente R, Andriulli A: Amoxicillin/tetracycline combinations are inadequate as alternative therapies for Helicobacter pylori infection. Helicobacter 2002;7:99-104.

7 Sanches B, Coelho L, Moretzsohn L, Vieira G Jr: Failure of Helicobacter pylori treatment af- ter regimes containing clarithromycin: new practical therapeutic options. Helicobacter 2008;13:572-576.

8 Taghavi SA, Jafari A, Eshraghian A: Efficacy of a new therapeutic regimen versus two routinely prescribed treatments for eradication of Helicobacter pylori: a randomized, doubleblind study of doxycycline, co-amoxiclav, and omeprazole in Iranian patients. Dig Dis Sci 2009;54:599-603.

9 Akyildiz M, Akay S, Musoglu A, Tuncyurek M, Aydin A: The efficacy of ranitidine bismuth citrate, amoxicillin and doxycycline or tetracycline regimens as a first line treatment for Helicobacter pylori eradication. Eur J Intern Med 2009;20:53-57.

10 Basu PP, Rayapudi K, Pacana T, Shah NJ, Krishnaswamy N, Flynn M: A randomized study comparing levofloxacin, omeprazole, nitazoxanide, and doxycycline versus triple therapy for the eradication of Helicobacter pylori. Am J Gastroenterol 2011;106:19701975.

11 Wang Z, Wu S: Doxycycline-based quadruple regimen versus routine quadruple regimen for rescue eradication of Helicobacter pylori: an open-label control study in Chinese patients. Singapore Med J 2012;53:273-276.

12 Nelson ML, Levy SB: The history of the tetracyclines. Ann N Y Acad Sci 2011;1241:17-32.

13 Agwuh KN, MacGowan A: Pharmacokinetics and pharmacodynamics of the tetracyclines including glycylcyclines. J Antimicrob Chemother 2006;58:256-265.

14 Ghotaslou R, Leylabadlo HE, Asl YM: Prevalence of antibiotic resistance in Helicobacter pylori: a recent literature review. World J Methodol 2015;5:164-174. 